\title{
Approach tendency and threat display as related to social status of Siamese fighting fish, Betta splendens
}

\author{
JANICE A. MELISKA \\ Western Illinois University, Macomb, Illinois 61455 \\ CHARLES J. MELISKA \\ Monmouth College, Monmouth, Illinois 61462 \\ and \\ KERMIT T. HOYENGA, KATHERINE B. HOYENGA, and ERIC F. WARD \\ Western Illinois University, Macomb, Illinois 61455
}

\begin{abstract}
Forty-eight male Betta splendens were assigned to three groups of 16 (eight pairs) each. In two of the groups, pairs of fish were first allowed to fight until a dominance-subordinance relationship was established. Then each subject was tested on measures of time spent in approach and threat display toward either (1) its own pair member, with which it was experienced and familiar (Group EF), or toward (2) another combat-experienced fish, with which it was unfamiliar (Group EU). A third group of inexperienced and unfamiliar individuals (Group IU) were first exposed to their own mirror images and then tested for approach and threat-display duration. Pairs of these fish were then allowed to fight until dominance was determined. The three major findings were: (a) Dominant subjects approached and displayed significantly more than subordinates in all three groups; (2) Dominant subjects of Group IU did not differ significantly from dominants in the other two groups with respect to time spent in approach and display; and (3) Fish exposed to mirrors prior to combat required significantly fewer sessions to establish dominance than fish not receiving mirror exposure. Results suggest that dominant fish can be distinguished on the basis of their approach tendency and threat-display scores prior to actual physical combat. An habituation model of dominance determination was proposed.
\end{abstract}

The threat display of the male Siamese fighting fish, Betta splendens, is characterized by extension of the gill covers, erection of the fins, a deepening of body color, and a sequence of vibrating body movements (Simpson, 1968). This threat display is reliably emitted whenever a male encounters an unfamiliar male conspecific, and it will even occur with considerable vigor in response to a male model, or in response to the male's own mirror image (Johnson, 1972). Furthermore, Bettas will perform an instrumental response for the opportunity to display to a mirror image (Thompson, 1963) or to male models dissimilar to their own body coloration (Grabowski \& Thompson, 1968; Thompson \& Sturm, 1965).

When two male Bettas are confined in a small tank, they will fight until one establishes dominance over the other. Baenninger (1970) investigated the effect of prior combat experience on the behavior of Siamese fighting fish. Combat experience consisted of placing fish pairs together to fight until a dominance-subordinance relationship had been established; i.e., until the subordinate consistently fled at the approach of the

Supported by a research grant from the Western Illinois University Foundation. We thank Margaret Hastings for her illustration in Figure 1 and $A$. Dean Wright and William Hastings for their critical comments. Requests for reprints should be sent to Charles J. Meliska, Department of Psychology, Monmouth College, Monmouth, Illinois 61462 . dominant. These animals were then compared on their operant rates for mirror presentation. Dominants responded more frequently than subordinates for the opportunity to display to their own mirror images.

The present experiment was designed to study the effects of combat experience on Betta's tendency to approach and display toward another fish. The combat experience was comparable to that of Baenninger (1970). It was expected that fish which had established dominance in paired encounters would, thereafter, continue to approach and display more toward their respective subordinates than would subordinates toward their respective dominants. Furthermore, it was predicted that fish which had had the experience of establishing dominance over one fish would tend to approach and display more than subordinates, even in encounters with unfamiliar fish, so long as they were tested against fish which had previously proved to be subordinate. In other words, it was expected that, as a consequence of having established dominance in one encounter, a fish would have learned to approach and threaten subordinates, a response which would thereafter generalize even to unfamiliar subordinate fish.

Finally, it was expected that fish which were socially inexperienced (that had not engaged in recent aggressive combat) would not differ from one another in their tendencies to approach and display. 


\section{METHOD}

Subjects

Twenty-four pairs of male Siamese fighting fish, approximately 9 months of age when purchased from a tropical fish wholesaler, were randomly assigned to one of three groups, eight pairs to a group. Members of a pair were matched for size. Animals were individually housed and visually isolated from each other in 1-liter opaque jars maintained at a water temperature of $26^{\circ} \mathrm{C}-27^{\circ} \mathrm{C}$. Animals were kept in aged tap water treated with sulfathiazol for disease prevention. A 14-h light-10-h dark cycle was in effect. Subjects were fed freeze-dried brine shrimp once each morning. All animals received at least a 24 -h adaptation period to their home environment before beginning the experimental procedure.

\section{Procedure}

Three groups of fish were tested. In Group EF (socially experienced and familiar), pairs were first put together in a 4-liter glass tank filled three-quarters with aged tap water and illuminated by a $25-W$ overhead lamp. Each pair was placed into the tank for $15 \mathrm{~min}$ daily until a dominance-subordinance relationship was established. The tank was filled with aged tap water before each 15 -min encounter. Testing was terminated when one fish consistently fled at the approach of the other on 2 consecutive test days. Social experience was limited to $15 \mathrm{~min}$ daily because the experimenters had observed from previous work that allowing paired fish to establish dominance in one long bout often led to serious injury to one or both members. The pairs in Group EF required a mean of 4.1 15-min sessions to establish dominance.

On the following day, these same pairs were placed into the test apparatus (Figure 1), one fish into each of the two opposite compartments. The sliding door to the test subject's compartment was then removed to allow the test subject to swim about freely. A test subject was observed for time spent in approach and aggressive display for $15 \mathrm{~min}$. Approach scores represented the time the test subject spent within the markers $61 / 2 \mathrm{~cm}$ in front of the confined fish's compartment. Aggressive display was measured by the time the test subject spent in gill extension when it was within the markers. Time spent in aggressive display by the confined fish was also measured when the test subject was within the markers. Aged tap water was used to fill the apparatus for each test. Each member of a pair served once as the test fish and once as the confined fish for its respective pair member, using a counterbalanced order of testing.

Animals in Group EU (socially experienced and unfamiliar),

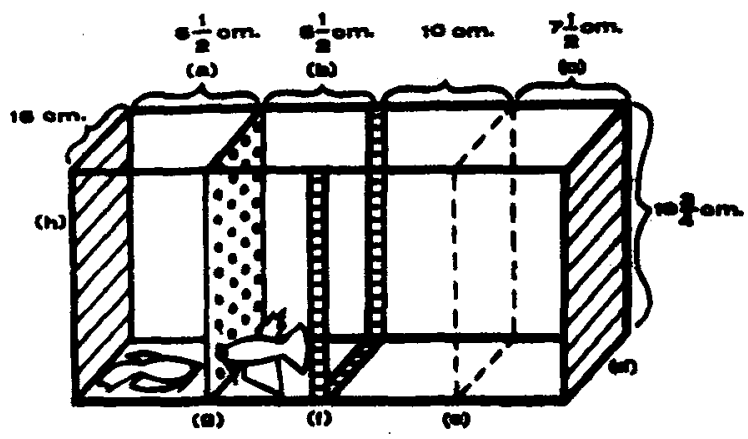

Figure 1. Diagram of the approach tendency and threat-display test apparatus. (a) Confined-subject compartment. (b) Approach area. (c) Test-subject compartment. (d), (h) White background. (e) Removable transparent door. (f) Tape marker. (g) Transparent partition drilled with $1 / 2-\mathrm{cm}$ holes for water circulation.

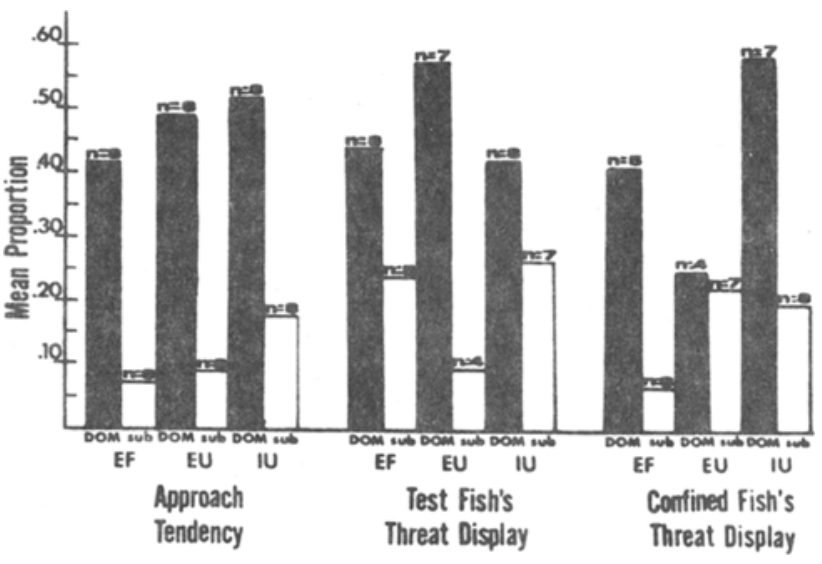

Figure 2. Mean proportional time recorded on three dependent variables by group (EF, EU, and IU) and by subjects' status (dominant or subordinate).

like those in Group EF, received combat experience first. Dominance was established in a mean of 4.215 -min sessions. The resulting dominants and subordinates were reassigned to new dominant-subordinate pairs. These unfamiliar pairs were then tested for approach and aggressive-display duration according to the same procedure described above.

Animals in Group IU (socially inexperienced and unfamiliar) did not immediately receive combat experience as had the animals in the other two groups. Instead, each subject was placed into a 1-liter opaque jar containing a $5 \times 5 \frac{1}{2} \mathrm{~cm}$ mirror, for 15 min daily. Animals were presented with the mirror for a mean of $3.915-\mathrm{min}$ exposures which approximated the 4.1 and 4.2 mean sessions required by the other two groups for social experience. In this way, the fish in Group IU were permitted to engage in display activities to at least a mirror image while the other animals displayed to and fought their pair members. Following mirror experience, the unfamiliar pairs of Group IU were tested for time spent in approach and aggressive display. On the next day, these same pairs were finally put together to establish dominance. Dominance was established in a mean of 2.515 -min sessions.

\section{RESULTS}

Figure 2 presents mean proportional time spent in approach and in aggressive display by test fish and confined fish. Approach proportions were calculated by dividing approach times by the 15 -min trial time. Aggressive-display proportions were calculated by dividing time spent in aggressive display by time spent in approach by the appropriate test subject.

Proportional-approach scores were analyzed by a 3 by 2 (EF, EU, and IU by Dom vs. Sub) factorial analysis of variance. The status factor was significant $(F=32.98$, $\mathrm{df}=1 / 42, \mathrm{p}<.001)$ but the group factor and the interaction were not significant at the .05 level, indicating that dominant subjects spent more time near subordinates than vice versa, regardless of group experience.

Proportional time spent in aggressive display by test subjects was analyzed by a 3 by 2 (EF, EU, and IU by 
Dom vs. Sub) unweighted means analysis for samples with unequal ns. (A conservative approach was taken in analyzing these data by omitting "zero" display scores made by test subjects which spent no time in approach.) Only the status factor was significant $(F=4.15$, $\mathrm{df}=1 / 33, \mathrm{p}<.05)$. Dominant test subjects spent $a$ greater proportion of their time in aggressive display than did subordinate subjects. The group factor and the interaction were not significant.

The same analysis was used to analyze the proportion of time that confined fish spent in aggressive display. Again, only the status factor was significant $(F=4.60$, $\mathrm{df}=1 / 33, \mathrm{p}<.05)$. Confined dominant subjects spent a greater proportion of time in aggressive display than did confined subordinates.

Whether dominants differed from subordinates with respect to certain physical characteristics such as color and size was also investigated. The frequencies with which a particular color was associated with dominance and subordinance were analyzed by a chi-square test modified by Yates correction for small expected frequencies (Guilford, 1965). No significant differences were found. An analysis of subject length in centimeters from mouth to juncture of body and tail was performed using a 3 by 2 (EF, EU, and IU by Dom vs. Sub) factorial analysis of variance. Again, no significant differences were found.

Since color and size were unrelated to dominance or subordinance, an attempt was made to predict individuals' social status from the approach duration and aggressive-display data of subjects in Group IU. As described previously, these fish were first exposed to mirror images and then tested for approach tendency. For one pair, no prediction as to dominance could be made. In six of the other seven pairs, we correctly predicted that the fish judged to display most avidly to a mirror (a forced choice based on color intensity, frequency of display, etc.) would dominate its pair member when given the opportunity.

As for the data on approach duration, we predicted that the animal which spent the greater proportion of time in approach would dominate the pair member which spent the smaller proportion in approach. We were correct in seven out of eight cases.

From the aggressive display data, it was predicted that the test subjects which spent the greater proportion of time in threat display, as compared to their pair members, would be dominant. In two cases, the pair members' proportions were equal, so no prediction could be made. In the other six cases, dominance was predicted correctly. [Baenninger (1968) found that those fish which displayed most frequently to a mirror image, whether under display-suppression drug conditions or nondrug conditions, were dominant in later group encounters.]

\section{DISCUSSION}

As predicted, dominant animals approached and spent more time near their respective subordinates than the subordinates spent near their respective dominants (see Figure 2). In addition to the approach-duration data, proportional times spent in aggressive display by test subjects and by confined subjects were also recorded. In both cases, dominant subjects spent significantly more time in aggressive display near subordinates than vice versa, regardless of their previous experience. This unexpected result indicates that time spent in aggressive display and approach were not influenced by recent combat nor by the familiarity of members during the approach test.

The results clearly show that neither familiarity with an opponent nor the recent experience of establishing a dominance relationship is necessary for differences in approach and display durations to appear. Figure 2 shows that dominants exceeded subordinates, across groups, on all three dependent measures taken: approach tendency, confined fish display, and test-fish display. Dominant fish always exceeded subordinates on these dependent measures regardless of whether fish had received combat experience immediately prior to the test. Thus, the social status of the animals in Group IU was strikingly apparent even before they were put together to establish a dominance relationship.

The question then arises, what characteristics distinguish a potential dominant from a potential subordinate? Body color was found to be unrelated to social status, nor did dominant animals differ significantly from subordinates on the basis of size. It is likely that a Betta's social background, especially its history of wins over losses to an opponent, influences its later behavior toward another animal. The fish used in the present experiment were allegedly raised separately (by a commercial breeder) once the males had become sexually dimorphic from the females. All subjects used were free of the fin scar tissue resulting from previous fighting encounters with other fish. However, the possibility remains that the potential dominants in Group IU had early social experiences differing from those of the potential subordinates. Except for Braddock and Braddock (1958), research is lacking on the relationship between early social experience and later behavior of the Siamese fighting fish.

Dominance may also depend upon the rapidity with which threat behaviors habituate in a particular fish. Habituation has been defined as a change, usually a reduction in responding, associated with repeated presentations of a stimulus (Ratner, 1970). This change in responding has been considered to be a relatively long-term effect which distinguishes habituation from adaptation, a short-term change in responding (Ratner, 
1970; Peeke and Peeke, 1973). The habituation of aggressive responding has been discussed by Peeke and Peeke (1973), by Peeke, Herz, and Gallagher (1971) and by van den Assem and van der Molen (1969) in terms of its survival value. In sum, these investigators have suggested that habituation suppresses aggressive tendencies in territorial fish so that they can respond to other stimuli necessary for their own survival as well as for their species.

Thus the habituation of aggressive responding may play an important role in the attenuation of conspecific aggression. Additionally, habituation may play an important role in the determination of dominance in Siamese fighting fish. The threat display of the Betta habituates upon repeated exposure to the image of another Betta whether it be its own mirror image (Clayton \& Hinde, 1968; Shapiro \& Schuckman, 1971) or that of a live conspecific (Baenninger, 1966; Figler, 1972; Peeke \& Peeke, 1970). Figler and Klein (Note 1) found that when paired Bettas were separated by a transparent partition for 6 consecutive hours, responding attenuated to such a degree that they would not fight one another when given the opportunity.

The habituation of aggressive responding may serve as the basis for the establishment of dominance. Any visual encounter between two fish provides the conditions for the elicitation and subsequent habituation of aggressive responding by both individuals. Continued exposure would be expected to cause a reduction in approach and threat behaviors by both fish. Fish whose aggressive responding habituates at a slow rate should dominate fish whose aggressive responding habituates rapidly. In other words, fish that persist in aggressive responding the longest will tend to become dominant over fish that are less persistent.

This hypothesis is supported by the data concerning duration of exposure required to establish dominance by the various groups in the present experiment. Fish allowed to fight first, prior to being tested for approach and threat-display duration (Groups EF and EU), required, on the average, 4.1 and 4.215 -min sessions, respectively, to establish dominance. On the other hand, Group IU fish which were given mirror exposure for a mean of 3.915 -min sessions plus the approach and threat-display test, prior to being tested for dominance, required a mean of only 2.5 sessions to establish dominance-significantly less time $(p<.05)$ than the two groups which did not receive this exposure prior to the test for dominance. This result is consistent with the expectation that the establishment of dominance is hastened by the habituation of approach and threat-display behaviors prior to combat.

Although the data cited above are consistent with the habituation hypothesis, they provide only indirect support for the hypothesis. Also, any causative relationship between habituation and social status could be objected to on the grounds that the habituated response may be stimulus specific as suggested in the work of Baenninger and Mattleman (1973), Peeke and Peeke (1973), and Gallagher, Herz, and Peeke (1972). However, it is likely that a certain amount of generalization occurs between similar stimuli. The problem then is to construct a situation that allows the effects of habituation to be expressed in observable behavior. For example, van der Assem and van den Molen (1969) found that in the three-spined stickleback, the habituation of responses to one opponent generalized to a new opponent when intraterritorial aggressive behavior was investigated rather than boundary fighting.

The habituation hypothesis and the assumption of stimulus generalization could be tested directly by pairing fish which have had repeated visual exposure to another Siamese fighting fish (the habituation group) with fish which have had no such exposure. If the individuals in the habituation group prove to be subordinate to nonhabituated controls, then there is compelling evidence for accepting habituation as an important factor in the determination of social dominance.

In conclusion, the present experiment provides a means for predicting the relative dominance of paired Siamese fighting fish on the basis of some observable behavioral characteristics. The rate of habituation of aggressive responding to aggressive stimuli was suggested as one of the factors that may determine dominance.

\section{REFERENCES}

Assem, J. van den, \& Molen, J. N. van der. Waning of the aggressive response in the three-spined stickleback upon constant exposure to a conspecific. I. A preliminary analysis of the phenomenon. Behaviour, 1969, 34, 286-324.

Baenninger, $R$. Waning of aggressive motivation in Betta splendens. Psychonomic Science, 1966, 4, 241-242.

Baenninger, $R$. Catechol amines and social relations in Siamese fighting fish. Animal Behaviour, 1968, 16, 442-447.

Baenninger, $R$. Visual reinforcement, habituation and prior social experience of Siamese fighting fish. Joumal of Comparative and Physiological Psychology, 1970, 71, 1-5.

Baenninger, R.. \& Mattleman, R. A, Visual reinforcement: Operant acquisition in the presence of a free mirror. Animal Learning and Behavior, 1973, 1, 302-306.

Braddock, J. C., \& Braddock, Z. I. Effects of isolation and social contact upon the development of aggressive behavior in the Siamese fighting fish. Animal Behaviour, 1958, 6, 249 (abstract).

Clayton, F. L., \& Hinde, R. A. The habituation and recovery of aggressive display in Betta splendens. Behaviour, 1968, 30, 96-106.

Figler, M. H. The relation between eliciting stimulus strength and habituation of the threat display in male Siamese fighting fish. Behaviour, 1972, 42, 63-96.

Gallagher, J. E., Herz, M. J., \& Peeke, H. V. S. Habituation of aggression: The effects of visual social stimuli on behavior between adjacently territorial convict cichlids (Cichlasoma nigrofasciatum). Behavioral Biology, 1972, 7, 359-368.

Grabowski, J. G., \& Thompson, T. Effects of visual reinforcer brightness and color on operant behavior of Siamese fighting fish. Psychonomic Science, 1968, 11, 111-112.

Guilford, J. P. Fundamental statistics in psychology and education. New York: McGraw-Hill, 1965, 237-238.

Johnson, R. N. Aggression in man and animals. Philadelphia: Saunders, 1972 . 
Peeke, H. V. S., Herz, M. J., \& Gallagher, J. E. Changes in aggressive interaction in adjacently territorial convict cichlids A study of habituation. Behaviour, 1971, 40, 43-54.

Peeke, H. V. S., \& Peeke, S. C. Habituation of conspecific aggression responses in the Siamese fighting fish. Behaviour, $1970,36,232-245$.

Peeke, H. V. S., \& Peeke, S. C. Habituation in fish with special reference to intraspecific aggressive behavior. In $H, V$. S Peeke and M. J. Herz (Eds.), Habituation I: Behavioral studies. New York: Academic Press, 1973.

Ratner, S. C. Habituation: Research and theory. In J. $H$. Reynierse (Ed.), Current issues in animal learning: $A$ colloquium. Lincoln: University of Nebraska Press, 1970.

Shapiro, S., \& Schuckman, H. Habituation and covariation of the components of the threat display in Betta splendens. Psychological Reports, 1971, 28, 827-837.

Simpson, M. J. A. The display of the Siamese fighting fish, Betta splendens. Animal Behaviour Monographs, 1968, 1, 1-73.
Thompson, T. I. Visual reinforcement in Siamese fighting fish. Science, $1963,141,55-57$.

Thompson, 'T., \& Sturm, T. Visual-reinforcer color and operant behavior in Siamese fighting fish. Journal of the Experimental Analysis of Behavior, $1965,8,341-344$.

\section{REFERENCE NOTE}

Figler, M. H.; \& Klein, R. M. Habituation of the appetitive components attenuates subsequent consummatory components of the agonistic sequence in male Siamese fighting fish (Betta splendens). Paper presented at the meeting of the Animal Behavior Society, Urbana-Champaign, Illinois, May 1974.

(Received for publication August 20, 1974; revision received December 2,1974 .) 